\title{
THE RESCUE OF AN INSURANCE COMPANY AFTER RUIN
}

\author{
KARL BORCH
}

Bergen

\section{INTRODUCTION}

1.1. In the different versions of the "Theory of Risk" it is almost universally assumed that ruin or bankruptcy marks the end of the game. The earlier versions of the theory tried to estimate the probability of this event, and studied the steps which an insurance company could take to bring probability of ruin down to an acceptable level. The more modern versions of the theory of risk tend to formulate the problem in economic terms, and study the cost of postponing or avoiding ruin.

In a recent discussion of a paper [4] surveying the development of the theory of risk, Professor Bather suggested that ruin may not necessarily be the end. If an otherwise sound insurance company gets into difficulties, so that ruin looms large, it is very likely that steps will be taken to rescue the company, for instance by refinancing, or in more extreme cases, by a merger.

1.2. To practical insurance men the simple suggestion of Professor Bather may seem next to trivial. Insurance companies get into difficulties fairly regularly, and rescue operations are considered in the insurance world, if not daily, at least annualy. The suggestion has, however, far-reaching implications for the theory of risk, and these do not seem to have been fully realised. If ruin does not mean the end of the game, but only the necessity of raising additional money, the current theories of risk may have to be radically revised. In this paper we shall discuss some of these implications.

\section{The Basic Model}

2.1. As our starting point we shall take a model due to de Finetti [5]. The model, which can be taken as the foundation of the modern theory of risk, can be defined as follows: 
(i) The company has an initial capital $S$.

(ii) In each operating period the company underwrites identical portfolios of insurance contracts. The profit of these portfolios is a stochastic variable with positive expectation, and with the distribution $F(x)$.

(iii) If at the end of an operating period the capital is negative, the company is ruined, and has to go out of business.

(iv) If at the end of an operating period the company's capital exceeds $Z$, the excess is paid out-as dividend or taxes, as the case may be.

In this model the company's capital performs a random walk. There is an absorbing barrier at $S=0$, and a reflecting barrier at $S=Z$. The amount $Z$ can be interpreted as the required reserve, i.e. as the reserve capital which the company considers necessary before any dividend can be paid.

2.2. The choice of a value $Z$ will determine a sequence of dividend payments: $s_{0}, s_{1}, \ldots, s_{t}, \ldots$, where $s_{t} \geq 0$ is the dividend paid at the end of the underwriting period $t$. For the expected discounted sum of these payments, we shall write:

$$
V(S, Z)=\sum_{t=0}^{\infty} v^{t} E\left(s_{t}\right)
$$

If they company is quite free in choosing $Z$, it is natural to assume that it will select the value of $Z$, which maximizes $V(S, Z)$. The assumption may, however, be unrealistic. It implies that neither government regulations nor pressure from the public will force the company to maintain larger reserves in order to provide more security to its customers. If we ignore these objections, we are led to a well defined mathematical problem, which as we shall see, has a considerable interest.

Before we attack this problem, a word of caution may, however, be useful. If the real objective of the company is to maximize the expected discounted value of its dividend payments, the real problem is to find the dividend policy, which will achieve this objective. A general dividend policy must specify the amount to be paid at the end of period $t$ in all conceivable circumstances. This means that the amount may depend on previous dividend payments $s_{0}, s_{1} \ldots s_{t-1}$, and on the company's past history, which 
in our model can be described by the sequence $S_{0}, S_{1} \ldots S_{t}$, the company's capital at the end of each previous underwriting period. The dividend policy is then defined by a function

$$
s_{t}=s\left(s_{0}, \ldots s_{t-1}, S_{0} \ldots S_{t}, t\right)
$$

The general problem consists in finding the best, or optimal dividend policy, when the objectives of the company are known. These objectives may be complex, they may for instance include a desire for a stable series of dividend payments, and for a steady growth of the company's capital.

2.3. If the company has the simple objective of maximizing the expected discounted value of the dividend payments, the optimal policy will be of a much simpler form. The payment at the end of period $t$ will then depend only on the actual state of the company at that time, i.e. the policy is defined by a function of the form

$$
s_{t}=s\left(S_{t}\right) \text {. }
$$

This result appears reasonable on intuitive reasons, and it has been proved rigorously by several authors, i.a. by Blackwell [I].

The dividend policy determined by a fixed reserve requirement $Z$ will be defined by a function

$$
\begin{array}{ll}
s(S)=0 & \text { for } S<Z \\
s(S)=S-Z & \text { for } Z<S
\end{array}
$$

This policy may, however, not be optimal. Morrill [6] has proved that when the distribution $F(x)$ is discrete and finite, the function defining the optimal policy is of the form

$$
\begin{array}{ll}
s(S)=0 & \text { for } S<Z_{0} \\
s(S)=S-Z_{0} & \text { for } Z_{0}<S<Z_{1} \\
. . . . . & . . . . . . . \\
s(S)=0 & \text { for } Z_{2 n-1}<S<Z_{2 n} \\
s(S)=S-Z_{2 n} & \text { for } Z_{2 n}<S
\end{array}
$$

This means that the function which defines the optimal policy is determined by a finite set of numbers $Z_{0}, Z_{1} \ldots Z_{2 n}$. Only if $n=0$, will this policy correspond to a fixed reserve requirement. 
2.4. Let us now put aside the difficulties indicated above, and determine the function $V(S, Z)$ defined at the beginning of para 2.2. It follows from the definition that we have

$$
\begin{array}{ll}
V(S, Z)=0 & \text { for } S<0 \\
V(S, Z)=S-Z+V(Z, Z) & \text { for } Z<S
\end{array}
$$

For $0 \leq S \leq Z$ we see that $V(S, Z)$ must satisfy the integral equation

$$
V(S, Z)=v \int_{-s}^{\infty} V(S+x) d F(x)
$$

or

$$
V(S, Z)=v \int_{z-s}^{\infty}\{x+S-Z+V(Z, Z)\} d F(x)+\int_{-s}^{z-s} V(S+x) d F(x)
$$

If the distribution $F(x)$ is discrete, this equation reduces to a difference equation. This case, which has been discussed by de Finetti [5] and Morrill [6], leads to some complications. It has been shown in another paper [3] that $V(S, Z)$ as function of $Z$ is discontinuous, and that as function of $S$ it does not have a continuous derivative.

If $F(x)$ is continuous, and a density function $f(x)=F^{\prime}(x)$ exists, the basic equation can be written

$$
V(S, Z)=v \int_{0}^{\infty}\{x+V(Z, Z)\} f(x-S+Z) d x+\int_{0}^{Z} V(x) f(x-S) d x
$$

This is an integral equation of Fredholm's type. It has a unique continuous solution given by the Neumann expansion

$$
V(S, Z)=\sum_{n=1}^{\infty} v^{n} \int_{0}^{\infty}\{x+V(Z, Z)\} f^{(n)}(x+Z-S) d x
$$

Here

$$
\begin{aligned}
& f^{(1)}(x-S)=f(x-S) \\
& f^{(n)}(x-S)=\int_{0}^{z} f^{(n-1)}(x-t) f(t-S) d t \quad n>\mathrm{I}
\end{aligned}
$$

This result can be verified by a direct probabilistic argument $f^{(n)}(x+Z-S) d x$ is the probability that the company shall have a capital $x+Z$ at the end of period $n$, provided that the company was neither ruined nor paid any dividend at the end of the $n-\mathbf{I}$ 
preceding periods. If the event $x>0$ occurs, the company will pay a dividend $x$, and the value of future dividend payments will be equal to $V(Z, Z)$. Since the event occurs at the end of period $n$, the expected discounted value of the payment is obtained by multiplying with $v^{n}$ and integrating over all non-negative $x$.

\section{Ruin And Rescue}

3.1. In the model we have outlined, we shall assume that the optimal dividend policy is determined by a single number $Z$, which we can interpret as the optimal reserve requirement. If the company's capital at the end of an underwriting period is $S<Z$, the expected discounted value of the future dividend payments is $V(S, Z)$. It is clear that $V(\mathrm{o}, Z)$ is positive. Even if the company has no capital (free reserves), there is a positive probability that a dividend will be paid before ruin. If $S<0$, we have, however, by definition $V(S, Z)=0$, because the company is not allowed to do any underwriting if its capital is negative. This means that if the company's capital falls from $\varepsilon$ to $-\varepsilon$, there will be a fall in dividend expectation from $V(\varepsilon, Z)$ to 0 ; a fall which may seem out of proportion to the actual loss of capital.

3.2. Let us now assume that the company at the end of an underwriting period is unable to pay the claims made against it, and let us assume that the deficit is $T$. The value of the company to the shareholders is then

$$
V(-T, Z)=\mathrm{o}
$$

since the company is not allowed to operate.

If the shareholders put up the money necessary to settle the claim, the company can continue its operations, and its value to the shareholders will be $V(0, Z)$. It will then be a good investment to rescue the company if

$$
V(\mathrm{o}, Z)-T>\mathrm{o}
$$

If the shareholders put up an amount of money $S$, in addition to the amount $T$ which is strictly necessary, their gain will be

$$
V(S, Z)-(S+T)
$$


They may then try to determine the value of $S$, which maximizes this difference, i.e. to find the optimal scheme for re-financing the company. If $V(S, Z)$ is differentiable with respect to $S$, this value is given by the condition

$$
\frac{\partial V(S, Z)}{\partial S}=\mathrm{I}
$$

It is fairly obvious that $S=Z$ will be a solution of this equation. Heuristically this result is obtained by the following argument:

If

$$
\frac{\partial V(S, Z)}{\partial S}>\mathrm{I}
$$

an additional amount of capital will increase the dividend expectations more if it is added to the reserves, than if it is paid out as dividend immediately. As $Z$ is the optimal reserve, it follows that the inequality must hold for all $S<Z$.

3.3. From the considerations in the preceding paragraph it follows that it is a good investment to rescue an insolvent insurance company, provided that its deficit is not too great. It is easy to see that the upper limit of the deficit is given by

$$
T<V(Z, Z)-Z
$$

If the deficit exceeds this limit, the shareholders should accept ruin, and let the company go into liquidation.

This result is not generally valid. There are a number of complications, particularly if the distribution $F(x)$ is discrete. These complications are, however, of a fairly trivial nature, and can best be illustrated by numerical examples, as we shall do in the following Section. The complications are due to the discontinuities of $V(S, Z)$ and its derivatives, and it is a tedious, but not very difficult task to sort them out.

3.4. We have so far taken an ad hoc approach to the rescue problem. We assumed that the company had already become insolvent, and we asked if it would be profitable for the shareholders to rescue the company. We based our analysis on the function $V(S, Z)$, and this is appropriate only if the company will have to go into 
liquidation the next time it becomes insolvent. If it is possible to rescue the company, also at the next crisis, $V(S, Z)$ will no longer represent the value of future dividend payments.

If the shareholders always are prepared to rescue the company, it is not necessary to keep any capital in the company. At the end of each operating period the owners will either divide the profits among themselves, or make a payment to cover the deficit. The expected profit in an arbitrary operating period is $E(x)$, and the discounted sum of these expected payments is

$$
W=\frac{v}{\mathbf{I}-v} \int_{-\infty}^{+\infty} x d F(x)
$$

It is obvious that $W \geq V(0, Z)$, since the obligation to cease operations after ruin cannot possibly increase the expected dividend payments. It is equally obvious that it does not pay to rescue the company if the deficit $T>W$. These considerations may lead the shareholders to seek some rule as to when they should cut their losses, and let the company go into liquidation. They may for instance decide that they will liquidate the company when the deficit becomes greater than $Y$. Let $W(Y)$ be the expected discounted value of the dividend payments (positive or negative), which will be made under this policy.

It is easy to see that $W(Y)$ must satisfy the equation:

$$
W(Y)=v \int_{-\mathbf{Y}}^{\infty}\{x+W(Y)\} d F(x)
$$

From this we find

$$
W(Y)=\frac{v}{\mathrm{I}-v+v F(-Y)} \int_{-Y}^{\infty} x d F(x)
$$

We can then determine the value of $Y$ which maximizes $W(Y)$. If a density function $f(x)=F^{\prime}(x)$ exists, this value is determined by the equation

$$
W^{\prime}(Y)=0
$$

or

$$
Y=\frac{v}{\mathrm{I}-v+v F(-Y)} \int_{-Y}^{\infty} x d F(x)
$$


This expresses the obvious. The company should be rescued only if the deficit is smaller than the expected profits from the rescue operation.

\section{Some Numerical Examples}

4.1. As our first example we shall take the simple case where $x$ can take only the values $-I$ and $+\mathrm{r}$. We shall assume

$$
\begin{aligned}
& \operatorname{Pr}(x=\mathrm{I})=p=0.565 \\
& \operatorname{Pr}(x=-\mathrm{I})=q=0.435
\end{aligned}
$$

The basic equation of para 2.4 is then reduced to the difference equation

$$
V(S, Z)=v p V(S+\mathrm{I}, Z)+v q V(S-\mathrm{I}, Z)
$$

This equation has been discussed in a number of other papers, i.a. [2] and [3]. It has the solution:

$$
V(S, Z)=\frac{r_{1}^{S+1}-r_{2}^{S+1}}{r_{1}^{Z+2}-r_{2}^{Z+2}-r_{1}^{Z+1}+r_{2}^{Z+Z}}
$$

where $r_{1}$ and $r_{2}$ are the roots of the characteristic equation

$$
r=v p r^{2}+v q \text {. }
$$

It is easy to verify that both roots are positive, and that $r_{1}>I$, $r_{\mathbf{2}}<\mathrm{I}$. It then follows that $V(S, Z)$ will increase with $S$ as we would expect. It also follows that $V(S, Z)$ will go to zero as $Z$ goes to infinity-i.e. if dividend payments are postponed indefinitely. If there is a value of $Z>0$, which maximizes $V(S, Z)$, this value

\begin{tabular}{|c|c|c|c|c|c|c|c|}
\hline \multirow{2}{*}{ 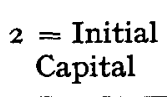 } & \multicolumn{2}{|c|}{$Z=$ Capital } & \multirow{2}{*}{$\begin{array}{c}\text { required } \\
2\end{array}$} & \multicolumn{2}{|c|}{ before dividends } & \multicolumn{2}{|c|}{ can be paid } \\
\hline & o & I & & 3 & 4 & 5 & 6 \\
\hline 0 & I. 25 & I. 49 & 1.70 & I. 83 & I. 89 & I. 88 & I. 82 \\
\hline I & 2.25 & 2.69 & 3.05 & $3 \cdot 3^{\circ}$ & $3.4 \mathrm{I}$ & 3.39 & 3.28 \\
\hline 2 & 3.25 & 3.69 & 4.19 & $4 \cdot 5^{2}$ & 4.67 & 4.66 & $4.5^{\circ}$ \\
\hline 3 & 4.25 & 4.69 & 5.19 & $5 \cdot 5^{6}$ & 5.79 & $5 \cdot 7^{6}$ & $5 \cdot 57$ \\
\hline 4 & 5.25 & 5.69 & 6.19 & 6.56 & 6.82 & 6.79 & 6.56 \\
\hline 5 & 6.25 & 6.69 & 7.19 & $7 \cdot 56$ & 7.82 & 7.78 & $7 \cdot 5 \mathrm{I}$ \\
\hline
\end{tabular}
will be independent of $S$, and can be taken as the "optimal" reserve.

\section{TABLE I}

$V(S, Z)=$ Expected discounted value of dividend payments 
If we take $v=0.983$, we find $r_{1}=$ I.I and $r_{2}=0.7$, and we can construct Table $\mathrm{I}$, which gives the value of $V(S, Z)$ for some selected values of $S$ and $Z$.

We see that in this example the optimal reserve is given by $Z=4$, at least as long as we only admit integral values of $Z$. To complete the analysis, we should of course investigate the meaning of nonintegral values of $Z$, but this is not necessary for our purpose.

In this simple discrete example, the only possible deficit is $T=$ I. If the capital of the company should be $-\mathrm{I}$ at the end of an underwriting period, the value of the company to the shareholders is zero. If, however, the shareholders put in new capital $T+Z=\mathrm{I}+4=5$, they get a refinanced company with the value $V(4,4)=6.82$, i.e. they make gain of $I .82$. This means that the ad hoc decision will be to raise money to refinance the company.

In this example the expected profit in an arbitrary operating period is $p-q=0.13$. If the shareholders always are ready to rescue the company from insolvency, the expected discounted value of the profit is

$$
W=\frac{v}{\mathrm{I}-v}(p-q)=6 . \mathrm{I} 5 .
$$

4.2. As our second example, we shall take another discrete case, which has been discussed in detail by Morrill [6]. We shall assume that:

$$
\operatorname{Pr}(x=\mathrm{I})=\mathrm{I} 2 / \mathrm{I} 3, \operatorname{Pr}(x=-2)=\mathrm{I} / \mathrm{I} 3, v=5 / 6
$$

Table II gives some values of $V(S, Z)$ for this example:

TABLE II

$$
V(S, Z)=\text { Expected discounted value of dividend payments }
$$

\begin{tabular}{cccccc}
\hline$S=$ Initial & \multicolumn{4}{c}{$Z=$ Capital required before } \\
Capital & \multicolumn{4}{c}{ dividends can be paid } \\
& o & r & 2 & 3 \\
\hline o & 5 & 4.17 & 4.89 & 4.23 \\
I & 6 & 5 & 5.87 & 5.24 \\
2 & 7 & 6 & 7.04 & 6.30 \\
3 & 8 & 7 & 8.04 & 7.21 \\
\hline
\end{tabular}


Here the optimal reserve seems to be $Z=2$. If the company holds a capital $S>2$, the expected discounted value of the dividend payments is maximized if the reserve requirement is set at $Z=2$, and the excess $S-Z$ is paid out. If, however, the company's capital should fall below this level, the company may set its reserve requirements lower. If the capital falls to $S=\mathrm{I}$, expected dividend payments will be $V(\mathrm{I}, 2)=5.87$, if the company maintains the reserve requirement $Z=2$. The company can, however, pay out a dividend of $I$, and continue its operations without any reserve capital. The expected dividend payments will then be $V(I, 0)=6$. Hence it will be profitable for the shareholders to lower the reserve requirement after an unfavourable operating period. It will, however, be even more profitable to refinance the company and bring its capital up to $S=2$.

In this example the greatest possible deficit is $T=2$. Should this occur, it will be a good investment for the shareholders to cover the deficit, and in addition bring the company's reserves up to the optimal level. The total outlay to the shareholders will be 4 , and in return they will get dividends with an expected discounted value $V(2,2)=7.04$.

4.3. As an example of a continuous distribution, let us assume that the density function has the following form:

$$
\begin{array}{ll}
f(x)=k \alpha e^{-\alpha x} & \text { for } x>0 \\
f(x)=(\mathrm{I}-k) \alpha e^{\alpha x} & \text { for } x<0
\end{array}
$$

In this case there is no upper limit to the company's gain, so the example may be a little unrealistic - at least in non-life insurance.

Suppressing the argument $Z$, we can write the integral equation of para 2.4 as follows:

$$
\begin{aligned}
V(S)= & v(\mathrm{I}-k) \alpha e^{-\alpha S} \int_{0}^{S} V(x) e^{\alpha x} d x \\
& +v k a e^{\alpha S} \int_{S}^{z} V(x) e^{-\alpha x} d x \\
& +v k V(Z) e^{\alpha(S-Z)}+\frac{v k}{\alpha} e^{\alpha(S-Z)}
\end{aligned}
$$

Differentiating twice, we find that the integral equation can be reduced to the differential equation 


$$
(\mathrm{I}-v) \alpha^{2} V(S)+v(\mathrm{I}-2 k) \alpha V^{\prime}(S)-V^{\prime \prime}(S)-V^{\prime \prime}(S)=0
$$

which has the general solution:

$$
V(S)=C_{1} e^{r_{1} S}+C_{2} e^{r_{2} S}
$$

Here $r_{1}$ and $r_{2}$ are the roots of the characteristic equation

$$
r^{2}-v(\mathrm{I}-2 k) \alpha r-(\mathrm{I}-v) \alpha^{2}=0
$$

and the constants $C_{1}$ and $C_{2}$ must be determined so that the general solution of the differential equation also is a solution of the integral equation.

Taking $\alpha=\mathrm{I}, v=0.97$, and $k=0.603$, we find:

$$
V(S, Z)=\frac{\mathrm{I} 43 e^{0.1 S}-9 \mathrm{I} e^{-0.3 S}}{\mathrm{I} 6 e^{0.1 Z}+2 \mathrm{I} e^{-0.3 Z}}
$$

Table III gives the value of $V(S, Z)$ for some selected values of $S$ and $Z$.

TABLE III

$V(S, Z)=$ Expected discounted value of dividend payments

\begin{tabular}{cccccccc}
\hline$S^{Z}$ & o & I & 2 & 3 & 4 & 5 & 6 \\
\hline o & I.4I & I.57 & I.68 & I.74 & I.73 & r.68 & I.6o \\
I & $2.4 \mathrm{I}$ & 2.74 & 2.93 & 3.02 & 3.02 & 2.94 & 2.70 \\
2 & $3.4 \mathrm{I}$ & 3.74 & 4.03 & 4.16 & 4.16 & 4.04 & 3.84 \\
3 & $4.4 \mathrm{I}$ & 4.74 & 4.03 & $5.2 \mathrm{I}$ & 5.20 & 5.14 & 4.88 \\
4 & $5.4 \mathrm{I}$ & 5.74 & 6.03 & $6.2 \mathrm{I}$ & 6.19 & 6.02 & 5.70 \\
5 & $6.4 \mathrm{I}$ & 6.74 & 7.03 & $7.2 \mathrm{I}$ & 7.19 & 6.98 & 6.60 \\
6 & $7.4 \mathrm{I}$ & 7.74 & 8.03 & $8.2 \mathrm{I}$ & 8.19 & 7.98 & 7.53 \\
\hline
\end{tabular}

It is easy to verify that $V(S, Z)$ has a maximum for $Z=3.45$, and that $V(3.45,3.45)=5.67$. In this example there is no limit to the deficit $T$, which can occur at the end of an operating period. From the result in para 3.3 it follows that it will be a good investment to rescue the company if

$$
T<V(Z, Z)-Z=5.67-3.45=2.22 \text {. }
$$

The argument in para 3.4 implies, however, that it will be profitable to rescue the company as long as the deficit $Y<6.65$. 4.4. It may be interesting to consider the probability of ruin in connection with the last example. If the company's capital 
is equal to the optimal reserve, i.e. $S=Z=3.45$, the probability that the company shall be ruined at the end of the next underwriting period, is

$$
F(-Z)=(\mathrm{I}-k) e^{-Z}=0.0 \mathrm{I}
$$

For the sake of argument we shall assume that this is accepted as adequate security for the policy holders. If the company's capital should fall to $S=\mathrm{I}$, the probability of ruin at the end of next period is

$$
F(-S)=(\mathrm{I}-k) e^{-S}=\text { o.I, }
$$

which is far less acceptable. According to the orthodox theory of risk, the company should now seek a reinsurance arrangement, which will bring the probability of ruin down to an acceptable level. This probability is, however, irrelevant. The security of the policy holders does not depend on the probability that the company shall become insolvent, but on the probability that the company shall become so insolvent that it is not worth rescuing. With the argument of para 3.3 this probability is

$$
F(-S-T)=(\mathrm{I}-k) e^{-S-T}=0.0 \mathrm{I},
$$

which may be quite acceptable.

With the argument of para 3.4 this probability is

$$
F(-Y)=(\mathrm{I}-k) e^{-Y}=0.0005,
$$

which is even more acceptable.

\section{Concluding Remarks}

5.1. The more orthodox theories of risk have been criticized for many reasons. The criticism has not always been very articulate, and neither have the replies. The subject has been discussed by actuaries for decades, but practical insurance people seem to have taken little part in this discussion. They seem in general to have considered both the theory of risk and the discussion around it as irrelevant to the problems which have to be solved by an insurance company in the real world.

The point we have tried to make in this paper, is that most studies in the actuarial theory of risk ignore the economic 
facts of life. The real weakness of the theory seems to be Assumption (i) in para 2.I. This assumption implies that the company will attract business of the same quality, regardless of its financial situation. This is a most unrealistic assumption, but it does not seem easy to modify it without constructing a general theory for the insurance market. Practical insurance men seem well aware that the assumption is unrealistic. Insurance companies usually advertise that they hold large reserves, presumably to attract business. If an insurance company goes into liquidation, it is usually because it has lost the power to attract good business, and not that random fluctuations has brought it to insolvency.

5.2. It may be appropriate to conclude with a quotation from Adam Smith: "... every individual is ... led by an invisible hand to promote an end which was no part of this intention. By pursuing his own interest, he frequently promotes that of the society more effectually than when he really intends to promote it". ([7], Book IV, Chapter 2).

When an actuary tries to apply the theory of risk, he usually intends to see that the customers of the insurance company obtain adequate security. Our examples indicate that the "invisible hand" may lead the actuary to do a better job of this, if he looked after the profits of his employers - the owners of the company.

\section{REFERENCES}

[I] Blackwell, D.: “Discrete Dynamic Programming", The Annals of Mathematical Statistics, Vol. 33 (1962), pp. 719-726.

[2] Borch, K.: "Control of a Portfolio of Insurance Contracts", The ASTIN Bulletin, Vol. IV (I966), pp. 59-7I.

[3] Borch, K.: "A Utility Function derived from a Survival Game", Management Science, Series B, Vol. I2 (I966), pp. $287-295$.

[4] Borch, K.: "The Theory of Risk", (with discussion), Journal of the Royal Statistical Society, Series B, Vol. 29 (I967), pp. 423-467.

[5] FinetTi, B. DE: "Su una Impostazione Alternativa della Theoria Collettiva del Rischio", Transactions of the $X V$ International Congress of Actuaries, 1957, Vol. II, pp. 433-443.

[6] Morrill, J.: "One-Person Games of Economic Survival", Naval Research Logistics Quarterly, Vol. I3 (1966), pp. 49-69.

[7] SмIтн, A.: The Wealth of Nations, Edinburgh 1776. 